\title{
A Visitor's Hybrid Smart Guide Control System
}

\section{Cliff Orori Mosiori}

Department of Mathematics and Physics, Technical University of Kenya, P. O. Box $90420-80100$, Mombasa, KENYA

"Corresponding Contact:

Email: corori@tum.ac.ke

\begin{abstract}
Modern industrial society is filled with hybrid systems. The effectiveness of a control system is now a basic requirement in any institution. In general terms, hybrid systems are reactive systems that intermix discrete and continuous control components in which the discrete part of that control system makes the decision on behave for the whole designed system and further decides if it can allow it to switch to another set of control protocols when appropriate conditions are availed. The continuous part only functions according to a specified protocol. Hybrid systems are common models used in digital (logic) and continuous networks and devices. Digital control programs detect, control and supervise continuous and discrete systems using differential or difference equations. This principle was utilized in our design and reported thereof. Although this work developed a different method to deal with specific problems facing students at Technical University of Mombasa. An upgrade was recommended so as to extend the control system to the eyeimpaired students and visitors. Since the issue of optimal smart control systems cannot be ignored in the design of new control systems with today's requirements in our learning institutions, it was concluded that further research was necessary to bring about a fully reliable and well-developed control system able to use real time and real routes as they exist at the University ground. This work reports a hybrid control guide system designed and implemented at Technical University of Mombasa.
\end{abstract}

Key words:

Hybrid Systems; Control Systems; Discrete System Control; Continuous System Control; Bellman's Optimality Principle

\section{INTRODUCTION}

Nowadays control technology has made it easier. You find that even a modern houses uses a complex logical decision control units to enable it perform human-like activities. Complex chemical processing in many chemical plants employ highly sophisticated control systems to perfect their automation to achieve higher efficiency. This has been enabled by the use of 
digital computers which equally use a mixture of discrete and continuous system controls. Household electric appliances refrigerator, washing machine and electric kettle all include control systems of one kind or other. Thus control is crucial to our daily life. Control systems are not limited in applications. In fact, we need reliable and accurate control systems to rock and open a door, guide new visitors or mature students or control traffic in an institution. Just like a fast response control system that is required for a computer hard disk drive to work properly, intelligent control system are essential in large institutions to control certain specific areas for new students or visitors.

\section{THEORY}

Control systems has become the order of life and security. Performance of a control system is measured based on its steady state error, gain margin and phase margin. These three parameters forms a very significant criterion of determining the quality, functionality, performance index and optimality of any control device or system. Control systems can be grouped into two categories as one with a closed-loop system, or as one with open-loop system (Han \& Lim, 2010). One main difference between the two is that an open-loop system directly utilizes an actuating device to control the whole process without feedback while in a closed-loop system (Han \& Lim, 2010; Sloo etal., 2018), the difference between the process under control and the reference input is indirectly used to control the process. In the second category, the resulting instantaneous difference is continuously reduced. Therefore, it is in order to think of a control system as a system with feedback in today's world (Moslehi \& Kumar, 2010) and always use feedback to enable us to control the desired output and its accuracy. In general, performance index of a control system is taken as a function of error detected between the actual response and the ideal responses (Venkatakrishnan et al., 2013). Therefore, designing any control system must obey the Bellman's Optimality Principle which states "An optimal policy has the property that no matter what the previous decision (i.e. controls) have been, the remaining decisions must constitute an optimal policy with regard to the state resulting from those previous decisions".

As a new design hybrid systems are taking the center stage in control system in modern applications (Saini et al., 2018). For hybrid systems, the applications of Bellman's principle leads to Hamilton Jacobi Bellman (HJB) relaxed variation form model (Zhang et al., 2018). If the cost-to-go functions are time-dependent, then the HJB equation becomes;

$$
\min _{u \in U(x)}\left\{l(x, u, t)+\frac{\partial G^{*}}{\partial t}+\sum_{i=1}^{n} \frac{\partial G^{*}}{\partial x_{i}} f_{i}(x, u, t)\right\}=0
$$

where $G^{*}$ is a function simultaneously dependant on both distance, $x$ and time, $t$. Eq. (1) can also be derived using the Taylor expansion by treating distance $x$ and time $t$ as the variables, hence [9];

$$
\min _{u \in U(x)}\left\{l(x, u, t)+\sum_{i=1}^{n} \frac{\partial G^{*}}{\partial x_{i}} f_{i}(x, u, t)\right\}=-\frac{\partial G^{*}}{\partial t}
$$

It therefore generalizes to the Hamilton-Jacobi-Isaacs (HJI) equations [13]. Suppose it was a hybrid system arbitrary defined using a cost term of the form $l(x, u, v, t)$. Then, the 
swapping of the min and max operators resembles the definition of saddle points and as a consequence, the Hamilton-Jacobi-Isaacs (HJI) equations that will characterize saddle equilibria will take the form of :

$$
\begin{aligned}
& \min _{u \in U(x)} \max _{u \in V(x)}\left\{l(x, u, v, t)+\frac{\partial G^{*}}{\partial t}+\sum_{i=1}^{n} \frac{\partial G^{*}}{\partial x_{i}} f_{i}(x, u, v, t)\right\}=0 \\
& \min _{u \in V(x)} \max _{u \in U(x)}\left\{l(x, u, v, t)+\frac{\partial G^{*}}{\partial t}+\sum_{i=1}^{n} \frac{\partial G^{*}}{\partial x_{i}} f_{i}(x, u, v, t)\right\}=0
\end{aligned}
$$

Suppose the variables, $\mathrm{X}$, and $\mathrm{U}$, are both continuous in a fixed discrete positions. The Hamilton-Jacobi-Isaacs (HJI) equations still hold true and therefore, thier present form as expressed in Eq. (4) can still be used for any system that is approximated by fixed discrete positions. From Eq. (1) and using the linear part of a Taylor series expansion about point $x$ , the term $G^{*}\left(x^{\prime}\right)$ can be estimated to:

$$
G^{*}\left(x^{\prime}\right) \approx G^{*}(x)+\sum_{i=1}^{n} \frac{\partial G^{*}}{\partial x_{i}} f_{i}(x, u) \Delta t
$$

From which it approximates to its tangent plane at point $x$. Substitution of (4) and (3) into and further substituting yields;

$$
G^{*}\left(x^{\prime}\right) \approx \min _{u \in U(x)}\left\{l(x, u) \Delta t+\partial G^{*}(x)+\sum_{i=1}^{n} \frac{\partial G^{*}}{\partial x_{i}} f_{i}(x, u) \Delta t\right\}
$$

Subtracting the $G^{*}\left(x^{\prime}\right)$ from both sides of (6), results into Eq. (7) as;

$$
\min _{u \in U(x)}\left\{l(x, u) \Delta t+\sum_{i=1}^{n} \frac{\partial G^{*}}{\partial x_{i}} f_{i}(x, u) \Delta t\right\}=0
$$

By approximation, taking the limit as $\Delta t$ approaches zero and dividing by $\Delta t$ results into the $H J B$ equation which this work based its design on as;

$$
\min _{u \in U(x)}\left\{l(x, u)+\sum_{i=1}^{n} \frac{\partial G^{*}}{\partial x_{i}} f_{i}(x, u)\right\}=0
$$

Eq. (8) is the continuous-time $H J B$ equation version and together with specified boundary conditions that are time and distance bound, the specific final-stage cost can be sufficiently characterized. It is an important expression for designing a solution over a whole state space since solutions to the HJB equation yield optimal feedback plans to determine the designed 
system. It is unfortunate to note that the HJB equation cannot be solved analytically in most applications and settings and therefore, this work used design approach by incorporating numerical iteration method (Novozhenets \& Regelski, 2009).

The advancement of information communication technologies (Zheng et al., 2018; Sims \& Stanway, 2003; Kang et al., 2018) I control system is now providing a good platform and oppor-tunities (Yang et al., 2005) for a paradigm shift for energy networks herein referred to as analog control system (Tao et al., 2017; Son et al., 2011; Krishnakumar \& Goldberg, 1992) to digital controls system (Zou et al., 2018b; Saini et al., 2018; Zhang et al., 2018; Alhelou et al., 2018; Nagarajaiah et al., 2008; Zheng et al., 2018; Werth et al., 2016; Yang et al., 2005) through automated control systems (Sloo etal., 2018; Novozhenets \& Regelski, 2009; Zou et al., 2018b; Ono et al., 2007; Alhelou et al., 2018) to smart control systems (Han \& Lim, 2010; Sloo etal., 2018; Venkatakrishnan et al., 2013; Zou et al., 2018b; Zhang et al., 2018; Son et al., 2011; Krishnakumar \& Goldberg, 1992) by setting the foundations for new social concepts based on meaningful collaboration and active participations (Zhang et al., 2018; Nagarajaiah et al., 2008; Wang et al., 2012). They too provide the frameworks for attaining functional fully decentralized smart control systems which are automated systems that are inherently more dynamic, localized, resilient, flexible, scalable and adaptable to small unique security and guiding systems (Farraj et al., 2016; Alhelou et al., 2018; Tao et al., 2017; Teslyuk et al., 2018; Georg et al., 2012; Zheng et al., 2018). Decentralization of smart control systems concerns not only the physical systems (human) and control logic (machine) but also the control structure (algorithm) which provides the network infrastructure on which safety Management depends on (Farraj et al., 2016; Alhelou et al., 2018; Teslyuk et al., 2018). This path or route guidance is rarely examined by articles reporting on Smart Control System despite it being a significant design element required in the actual implementation stage (Teslyuk et al., 2018).

Our control design appears in a wide variety of applications and forms the heart of most hybrid system designs. However, in this design, the logic unit and continuous unit were designed separately and then combined together by the two interfaces. It is a model developed to analyze a number of interactions between logical elements, the University points and the new student/visitor by including concepts from differential geometry, differential and difference equations, optimal control, automata (programs), discrete event systems, data structures and computation algorithms. The theoretical model was scenariobased and used scenario-based models and automata modified complex processes.

\section{Methodology}

\section{Design}

In this work, a hybrid systems was viewed as a large collection of systems with capabilities comprising of a hierarchical structure that obeys logic decisions only as illustrated by figure1. The discrete event control system was designed to have finite state machines and use fuzzy logic (Zou et al., 2018b) while the bottom layer was designed to use continuous event system and equally accommodate the physical system, in this case, the new student or visitor. 


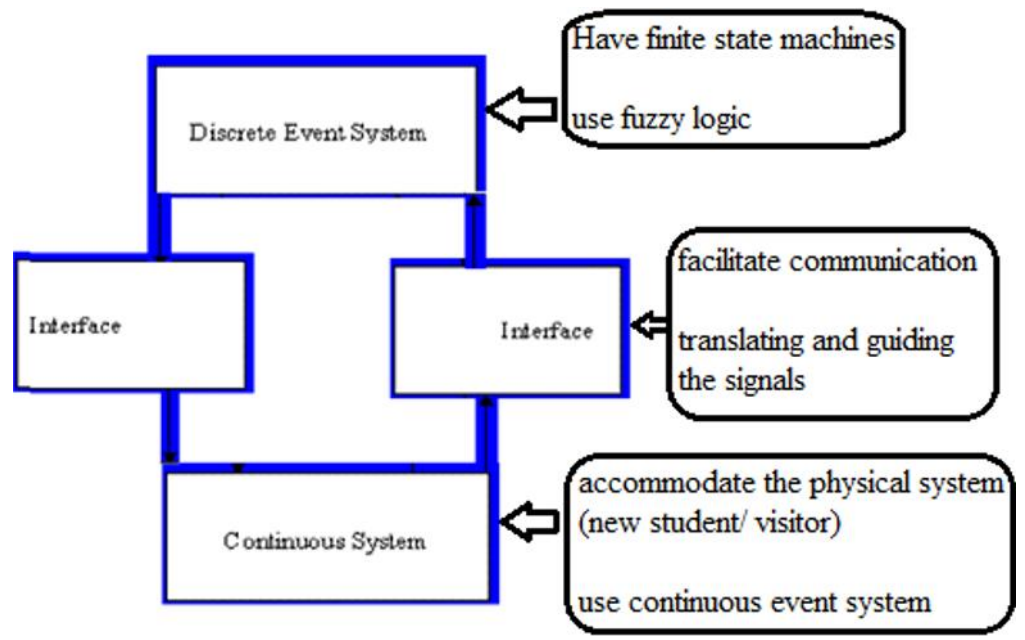

Figure 1: Logical obedience of Hybrid control System

\section{Interfaces}

The interfaces were designed to facilitate communication between the discrete event control system (points in the Technical University of Mombasa ground) and the continuous event system (new student/visitor) by means of translating and guiding signals between them. Each agent was structured and characterized by a model encoded in logic clauses (basically as If -Then$\underline{E l s e}$ algorithm) in hierarchy by enabling logic failures to trigger events, thus affecting the ongoing processes at that instant time. I real time, it enables the detection of logic failures and real time structural adaptation to rectify the processes.

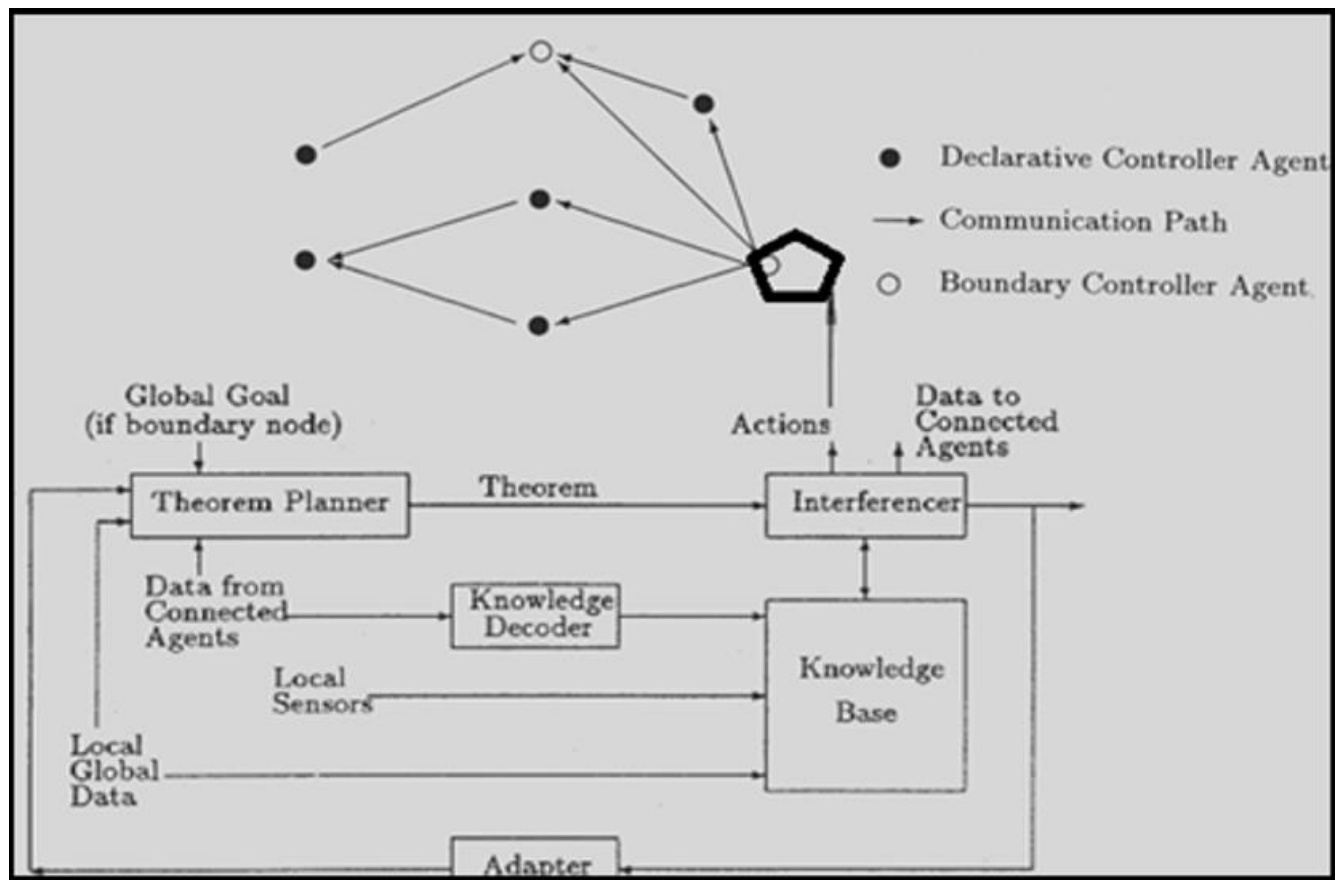

Figure 2: Network of cooperating control agents and areas of interest 


\section{Protocol}

A protocol algorithm was designed to enable to create or delete certain events from the network in real time. Components, sections, states or areas of the guide program that did not require a logic decision were modelled using continuous natural differential or difference equations derived from the physical world so that they can process continuous signals.

\section{Distributed Operating Commands}

A real-time distributed operating commands (Saini et al., 2018) were coded that could be generated by the agent programs, be sent to the system's controllers and actuators in real time and in real time, the data collected by the sensors is transmitted to the agent programs to generate well-accounted commands to accurately guide the course/ path/ route. The distances between all important points/ places /departments are shown in figure 3.

\section{Shortest Path Model}

A dynamic Programming was done in such a way that it first determines the optimal control state when there is only one stage left in the movement of the new student/ visitor and then determine the optimal control state when at least any two stages are left. The program included the state of continuing onwards and at some stage proceeds backwards if need be using the Shortest Path Problem Method based on linear time-varying systems and straightforward coding. In our case, if a new student or visitor wishes to determine the shortest path from Assembly Hall to a lecture Hall towards the east of the Assembly Hall while walking, the control system sends guiding signals appropriately to give direction, position and the expected time of arrival. The model paths in figure 3 were designed for purposes of testing the design. The control path and walk was designed and programmed to occur in three (3) major stages namely, A, B and C, and to end either in $\mathrm{N}_{c}, \mathrm{C}_{c}$ or Sc points or places.

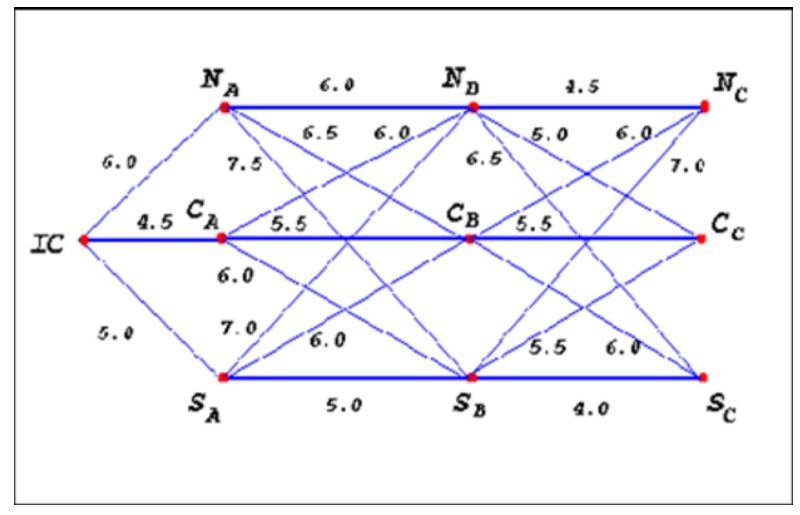

Figure 3: Model paths in TUM

\section{Path Control Algorithm}

The walking starts at the Assembly Hall (IC). The control system then exposes the new student or visitor to three new stages to choose from depending on what he/she needs. The

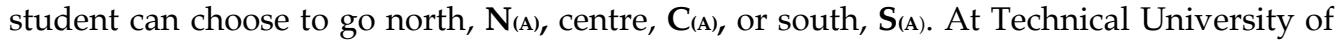
Mombasa, the Assembly Hall College is located near the main gate. If the students walks north for $60 \mathrm{~m}$, he will reach the gate $\mathbf{B}$ (position $\mathrm{N}_{A}$ ). If he/she walks for $45 \mathrm{~m}$ towards the 
center (straight), he reaches the Institute of Computing (ICI) (position $\mathrm{C}_{A}$ ). If he chooses to walk south for walking $50 \mathrm{~m}$, he/she will be at the faculty of Pure and Applied Sciences, Dean's office (position $S_{A}$ ) after. It means that the new student/visitor will either be in $\mathrm{N}_{A}$, $\mathrm{C}_{A}$ or $\mathrm{S}_{A}$ as the first new location (A) and after this, he has to decide which college he will travel to. The journey ends in stage $\mathrm{C}$ in either $\mathrm{N}_{c}, \mathrm{C}_{c}$ or $\mathrm{S}_{c}$.

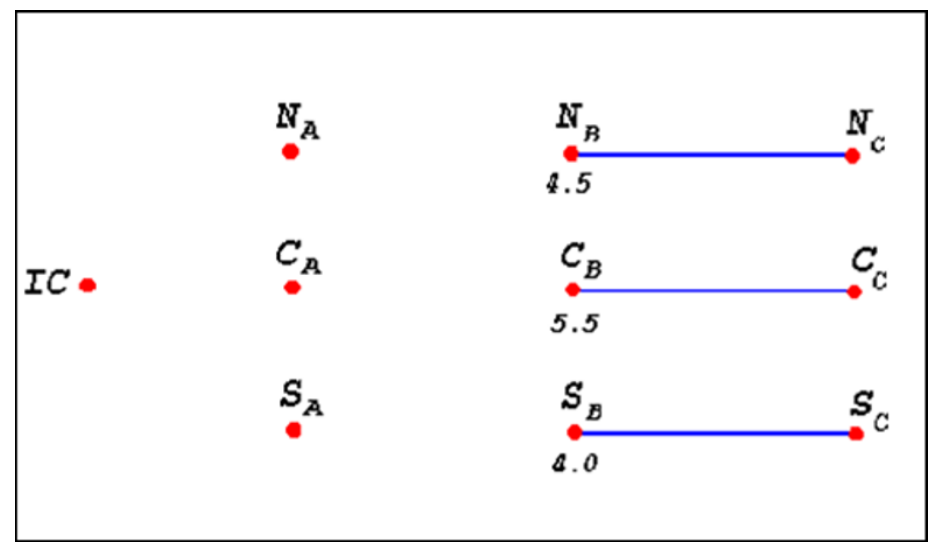

Figure 4: Choices of Paths

If the student/visitor walks and finds himself in $\mathrm{N}_{B}$, he has 3 choices; north, center or south, leading to $\mathrm{N}_{C}, \mathrm{C}_{c}$ or $\mathrm{S}_{c}$ respectively. It was programmed to go to $\mathrm{N}_{c}$ considering the distance. Similarly, if the students is in $C_{B}$, the best choice would be to go to $C_{C}$ for the same reason and if the student is in $S_{B}$, the best choice is to go to $S_{c}$. The next part of the algorithm was have the optimal decision when there are only 2 stages left. If the student is in $\mathrm{N}_{A}$ and walk north for $60 \mathrm{~m}$, he will reach $\mathrm{N}_{B}$ but required to walk a further $45 \mathrm{~m}$ to reach the Assembly Hall making a total journey of $105 \mathrm{~m}$ long.

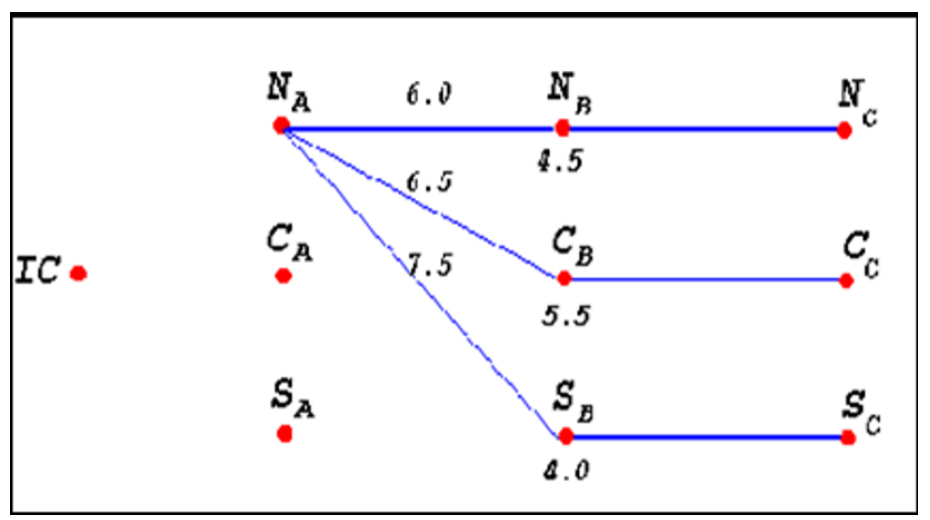

Figure 5: Choices of Paths

If he chooses to walk to towards the center of the University compound for $65 \mathrm{~m}$, he will reach $C_{B}$ which will require an additional $55 \mathrm{~m}$ to reach his final destination $120 \mathrm{~m}$ away. If he/she chooses south from $\mathrm{N}_{A}$ and walks for a total distance of $115 \mathrm{~m}$ to reach the gate $\mathrm{C}$ he/she will be exposed to three choices each with its own distance, choices and implication as illustrated in figure 5 and the optimal path from $C_{A}$ and $S_{A}$ as well as the shortest distance from these places to the Assembly Hall now has only two stages left is shown in figure 6 . 


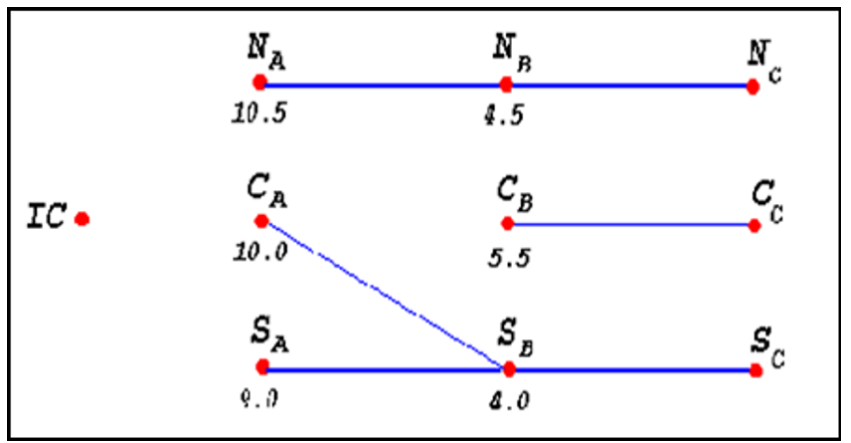

Figure 6: Final optimum paths

Finally, the student is in the Business department. If he goes north, he travels $60 \mathrm{~m}$ to reach $\mathrm{N}_{A}$ and then a further $105 \mathrm{~m}$ to reach the Assembly Hall and walks a total distance of $165 \mathrm{~km}$ or if he walks to the centre, he will travel a total distance of $145 \mathrm{~m}$ and a distance of $140 \mathrm{~m}$ if he goes south. Therefore the best choice is to travel south from business department and the total distance of his journey will be $14 \mathrm{~m}$ as illustrated in figure 4 .

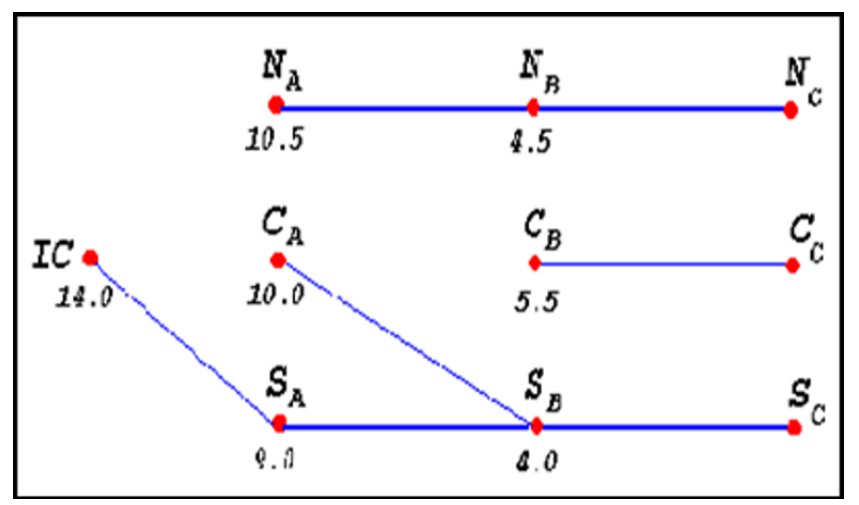

Figure 7: Final optimal path

\section{CONCLUSION}

A control system was designed and implemented at the Technical University of Mombasa to guide new students and visitors using a smart control guide. It was observed that in in order to walk the shortest path we actually solved the shortest path from all of the departments, $\mathrm{N}_{A}, \mathrm{C}_{A}, \mathrm{~S}_{A}, \mathrm{~N}_{B}, \mathrm{C}_{B}$ and $\mathrm{S}_{B}$ in Technical University of Mombasa. The outcome showed that programming can be computationally intractable if there are many stages and many possible decisions at each stage that easiness the process of determine the optimal logic decisions as a function of state and the number of stages. It also reaffirmed the confidence that using a closed-loop control design to determine the optimal solutions for ( $n$ +1 ) stages applying the backward recursion relationship forms a backbone programing condition in smart control systems. It was concluded that if one moves to state $x^{\prime}$ from $x$, then for optimal continuation decision making to the last destination using the shortest path from state $x^{\prime}$ to the end, each segments forms the next optimal paths and hence itself is an optimal point. This work developed confidence in the application of mathematical theory of multistage decision processes in control design undergraduate projects at the department of Mathematics and Physics of the Technical University of Mombasa. 


\section{REFERENCES}

Alhelou, H. H., Hamedani-Golshan, M. E., Zamani, R., Heydarian-Forushani, E., \& Siano, P. (2018). Challenges and opportunities of load frequency control in conventional, modern and future smart power systems: a comprehensive review. Energies, 11(10), 2497.

Farraj, A., Hammad, E., \& Kundur, D. (2016). A cyber-physical control framework for transient stability in smart grids. IEEE Transactions on Smart Grid, 9(2), 1205-1215.

Georg, H., Wietfeld, C., Müller, S. C., \& Rehtanz, C. (2012, November). A HLA based simulator architecture for co-simulating ICT based power system control and protection systems. In 2012 IEEE Third International Conference on Smart Grid Communications (SmartGridComm) (pp. 264-269). IEEE.

Han, D. M., \& Lim, J. H. (2010). Smart home energy management system using IEEE 802.15. 4 and zigbee. IEEE Transactions on Consumer Electronics, 56(3), 1403-1410.

Kang, C. W., Ramzan, M. B., Sarkar, B., \& Imran, M. (2018). Effect of inspection performance in smart manufacturing system based on human quality control system. The International Journal of Advanced Manufacturing Technology, 94(9-12), 4351-4364.

Krishnakumar, K., \& Goldberg, D. E. (1992). Control system optimization using genetic algorithms. Journal of Guidance, Control, and Dynamics, 15(3), 735-740.

Moslehi, K., \& Kumar, R. (2010, January). Smart grid-a reliability perspective. In 2010 Innovative Smart Grid Technologies (ISGT) (pp. 1-8). IEEE.

Nagarajaiah, S., Narasimhan, S., \& Johnson, E. (2008). Structural control benchmark problem: Phase II-Nonlinear smart base-isolated building subjected to near-fault earthquakes. Structural Control and Health Monitoring: The Official Journal of the International Association for Structural Control and Monitoring and of the European Association for the Control of Structures, 15(5), 653-656.

Novozhenets, Y., \& Regelski, M. (2009). U.S. Patent No. 7,475,812. Washington, DC: U.S. Patent and Trademark Office.

Ono, K., Kajiwara, I., \& Ishizuka, S. (2007). Piezoelectric and control optimisation of smart structures for vibration and sound suppression. International Journal of Vehicle Design, 43(1-4), 184-199.

Saini, S., Beniwal, R. K., Kumar, R., Paul, R., \& Saini, S. (2018). Modelling for improved cyber security in Smart distribution system. International Journal on Future Revolution in Computer Science $\mathcal{E}$ Communication Engineering, Accepted.

Sims, N. D., \& Stanway, R. (2003). Semi-active vehicle suspension using smart fluid dampers: a modelling and control study. International Journal of Vehicle Design, 33(1-3), 76-102.

Sloo, D., Webb, N. U., Fisher, E. J., Matsuoka, Y., Fadell, A., \& Rogers, M. (2018). U.S. Patent No. 9,905,122. Washington, DC: U.S. Patent and Trademark Office.

Son, J. Y., Park, J. H., Moon, K. D., \& Lee, Y. H. (2011). Resource-aware smart home management system by constructing resource relation graph. IEEE Transactions on Consumer Electronics, 57(3), 11121119.

Tao, F., Cheng, J., \& Qi, Q. (2017). IIHub: an industrial internet-of-things hub toward smart manufacturing based on cyber-physical system. IEEE Transactions on Industrial Informatics, 14(5), 2271-2280.

Taofik, A., Ismail, N., Gerhana, Y. A., Komarujaman, K., \& Ramdhani, M. A. (2018, January). Design of Smart System to Detect Ripeness of Tomato and Chili with New Approach in Data Acquisition. In IOP Conference Series: Materials Science and Engineering (Vol. 288, No. 1, p. 012018). IOP Publishing.

Teslyuk, V., Beregovskyi, V., Denysyuk, P., Teslyuk, T., \& Lozynskyi, A. (2018). Development and implementation of the technical accident prevention subsystem for the smart home system. International Journal of Intelligent Systems and Applications, 11(1), 1. 
Venkatakrishnan, N., Nolan, K. F., Bingham, D. C., \& Najewicz, D. J. (2013). U.S. Patent No. 8,560,133. Washington, DC: U.S. Patent and Trademark Office.

Wang, Z., Wang, L., Dounis, A. I., \& Yang, R. (2012). Multi-agent control system with information fusion based comfort model for smart buildings. Applied Energy, 99, 247-254.

Werth, A., André, A., Kawamoto, D., Morita, T., Tajima, S., Tokoro, M., ... \& Tanaka, K. (2016). Peer-topeer control system for DC microgrids. IEEE Transactions on Smart Grid, 9(4), 3667-3675.

Yang, Y., Jin, Z., \& Soh, C. K. (2005). Integrated optimal design of vibration control system for smart beams using genetic algorithms. Journal of Sound and Vibration, 282(3-5), 1293-1307.

Yoon, C., Huh, M., Kang, S. G., Park, J., \& Lee, C. (2018, February). Implement smart farm with IoT technology. In 2018 20th International Conference on Advanced Communication Technology (ICACT) (pp. 749-752). IEEE.

Zhang, Y., Zheng, D., \& Deng, R. H. (2018). Security and privacy in smart health: Efficient policy-hiding attribute-based access control. IEEE Internet of Things Journal, 5(3), 2130-2145.

Zheng, P., Sang, Z., Zhong, R. Y., Liu, Y., Liu, C., Mubarok, K., ... \& Xu, X. (2018). Smart manufacturing systems for Industry 4.0: Conceptual framework, scenarios, and future perspectives. Frontiers of Mechanical Engineering, 13(2), 137-150.

Zheng, X., Cai, Z., \& Li, Y. (2018). Data linkage in smart internet of things systems: A consideration from a privacy perspective. IEEE Communications Magazine, 56(9), 55-61.

Zou, H., Zhou, Y., Jiang, H., Chien, S. C., Xie, L., \& Spanos, C. J. (2018a). WinLight: A WiFi-based occupancy-driven lighting control system for smart building. Energy and Buildings, 158, 924-938.

Zou, H., Zhou, Y., Jiang, H., Chien, S. C., Xie, L., \& Spanos, C. J. (2018). WinLight: A WiFi-based occupancy-driven lighting control system for smart building. Energy and Buildings, 158, 924-938.

\section{ACKNOWLEDGEMENTS}

I wish to acknowledge and express sincere appreciation to mathematics and Physics department of Technical University of Mombasa for providing a testing ground for experimentation. I wish to appreciate Mr. Michael Rotich and Festus Rotich of the department of Electrical and Electronic Engineering, Rift Valley Technical Institute of Science and Technology, Mr. Bernard Nyakundi, Chairperson of Electrical and Electronic Engineering department, Mr. Valentine Wabwire Muramba of Mathematics and Physics department of Technical University of Mombasa respectively for taking their time to advice on this article. This article is a derived from a Bachelor of Technology in Applied Physics (Electronics and Instrumentation) Undergraduate project exhibited in April 2019 series Project Exhibition Day. 